\title{
Comments Concerning Measurements and Equations in Electromagnetism
}

\author{
Salama Abdelhady
}

Department of Mechanical Engineering, CIC, Cairo, Egypt.

E-mail: salama_hady@cic-cairo.com

Received November $10^{\text {th }}$, 2010; revised November 27 $7^{\text {th }}$, 2010; accepted December $2^{\text {nd }}, 2010$.

\begin{abstract}
The previously introduced US of units has raised many comments. Such comments were the claims of how to measure the electric current and magnetic flux. Other comments were concerned by violating the fundamental laws of electromagnetism. Such violation is not the concern of the introduced system of units. But it is related to the previous mess in the available SI system of units.
\end{abstract}

Keywords: Electric Charge, Magnetic Flux, Ampere's Law, System of Units

\section{Electric Charge}

Electric charge was defined, according to a previously introduced entropy-approach [1], as a form of energy or electromagnetic waves that have an electric potential, positive or negative. So; the unit of electric charge is a unit of Energy, i.e. in Joules. Hence; the current as a rate of flow of electric charge should be measured in Watt. Accordingly; the ammeter does not read such defined current but reads actually the rate of flow of electric energy per Volt. This can be shown from the following well known equation that is used in electric- power measurement or calculation [2]:

$$
\dot{W}_{e}=\mathrm{A} * \mathrm{~V} \cdots
$$

Where $\mathrm{A}$ can be defined as the ammeter reading and $\mathrm{V}$ is the potential difference.

Hence; the units of the Ammeter readings should be:

$$
\mathrm{A}=\frac{\dot{W}}{V} \cdots \frac{\text { Watt }}{\text { Volt }}
$$

So; the universal system of units, that was previously introduced [3], may use the same ammeter as an instrument for measuring the current in Watt/Volt. The Ammeter does not measure actually the rate of flow of electric energy as previously assumed in Coulomb/sec but it measures the flow of electric entropy associated by the electric energy [3].

\section{Magnetic Flux}

Magnetic Flux is defined also according to a published entropy approach and experimental work as a form of energy or electromagnetic waves that have magnetic potential, positive or negative [1]. So; the magnetic flux has to be measured in the same units of energy; i.e. in Joule. According to a scientific analogy between thermal, electric and magnetic energies and their common effect on an Al-Fe thermocouple [3]; it was found that their potentials could be measured also by the Volt. Revising the techniques of measurement of the magnetic flux; it is found that they depend on measuring the produced electric potential by the influence of the measured magnetic field on a conductor that carries an electric current [4]. So; the techniques used do not follow a direct approach but they depend on measuring electric-field parameters to find the corresponding magnetic parameters.

However; we can measure directly the magnetic energy that performs work in attracting iron balls along an inclined plane [1]. Hence; it is possible to recalibrate the already used Gaussmeter to measure the rate of flow of magnetic flux during such attraction process. According to the proved analogy between electric and magnetic fields and the common unit of their potentials as previously described; it is possible to assume the unit of measurement of the modified Gauss-meter to be identical to the unit of Ammeter readings; i.e. in Watt/volt. So; we may introduce the following equation to measure or calculate the magnetic power from readings of the Gaussmeter by an equation that is analogous to Equation (1):

$$
\dot{W}_{m}=G * H
$$


where $\mathrm{G}$ is the Gauss-meter reading and $\mathrm{H}$ is the magnetic potential in Volt. Hence; the unit of measurement in a modified Gauss-meter for measuring the rate of flow of magnetic flux per unit magnetic potential will be, according to Equation (3), as follows:

$$
G=\frac{\dot{W}}{V} \cdots \frac{\text { Watt }}{\text { Volt }}
$$

By analogy; this unit measures the rate of flow of magnetic entropy associated by the magnetic energy [3].

\section{Ampere's Law}

According to Ampere's law, the produced "E.M.F." or voltage at the open ends of the loop is dependent of the time rate of change of the magnetic flux encircled by the loop. According to electromagnetism References [5]; the found relation is expressed as follows:

$$
\frac{d \phi}{d t}=\mathrm{A} * \frac{d B}{d t}=-V
$$

Such equation proves that the units of magnetic and electric potentials are identical. However; substituting the SI units of the terms in sides of Equation (5); we find the following units will result:

$$
\mathrm{m}^{2} \frac{\mathrm{kg} / \mathrm{sec}^{2}}{A m b * \text { sec. }} \neq \text { Volt }
$$

Hence; this equation is incorrect from the dimensional point of view.

However; Ampere's law can be rewritten in adjustable dimensional form where the coefficient of proportionality between the E.M.F. or voltage at the open ends of the loop and the time rate of change of the magnetic flux encircled by the loop will be the loop area times the reluctance of the loop; i.e.

$$
\mathrm{A} * \mathrm{R}_{m} * \frac{d b}{d t}=-V
$$

According to the postulated US of units [3]; the units in both sides of Equation (6) will be equivalent:

$$
m^{2} * \frac{\text { Volt }}{W} * \frac{W}{m^{2}} \equiv \text { Volt }
$$

\section{REFERENCES}

[1] S. Abdelhady, "A Fundamental Equation of Thermodynamics that Embraces Electrical and Magnetic Potentials,” Journal of Electromagnetic Analysis \& Applications, Vol. 2, March 2010, pp. 162-168.

[2] Jewett, Jr. and R. A. Serway, "Physics for Scientists and Engineers with Modern Physics," 7th Edition, Thomson, 2008.

[3] S. Abdelhady, "An Approach to a Universal System of Units,” Jouranl Electromagnetic Analysis \& Applications, Vol. 2, March 2010, pp. 162-168.

[4] D. Gordon, R. Brown and J. Haben, "Methods for Measuring the Magnetic Field," Magnetics, IEEE Transactions, Vol. 8, No. 1, pp. 48-51.

[5] N. J. Carron, "On the Field of a Torque and the Observability of the Vector Potential," Physics Notes, Note 6, 1993. 